\title{
ON DUALITY IN PROXIMITY SPACES
}

\section{S. LEADER}

1. Introduction. Given a binary relation $\beta$ on a Boolean algebra $a$, define the dual relation $\beta^{*}$ to be the class of all ordered pairs $(P, Q)$ of elements of $Q$ such that $(P,-Q)$ does not belong to $\beta$. ( $-Q$ is the complement of $Q$.) The term "dual" is justified by the property, $\beta^{* *}=\beta$. Given a subclass $B$ of $Q$, one can identify $B$ with the universal relation whose domain is $Q$ and range is $B$. Motivated by this identification, define the dual class $B^{*}$ of $B$ to be the class of all elements of $a$ whose complements do not belong to $B$. Then

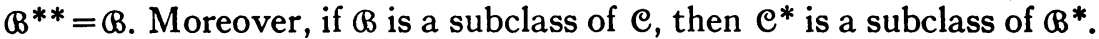

Now, for $X$ a proximity space [3] and $a$ the class of all subsets of $X$ these duality concepts are especially pertinent. The proximity relation $A \beta B$ (read " $A$ is close to $B$ ") on subsets of $X$ has as its dual the relation $A \ll B$ (read " $A$ is uniformly interior to $B$ " or equivalently, " $B$ is a uniform neighborhood of $A$ "). In terms of the proximity relation the simplest way to construct the Smirnov [10] compactification $\bar{X}$ is to identify $\bar{X}$ with the family of all clusters [7] from $X$. In terms of the dual relation the simplest way to construct the Smirnov compactification is to identify $\bar{X}$ with the family of all ends $[1 ; 2 ; 4 ; 5 ; 6]$ from $X$. Since the Smirnov compactification is unique, there is a oneone correspondence between clusters and ends. Our key result, proved without the compactification theorem, is that clusters and ends are dual classes. To expedite the proof of this duality (Theorem 10), we introduce a new definition of "end" which turns out (Theorem 9) to be equivalent to Alexandroff's definition [2]. Applying the duality theorem, we obtain a characterization (Theorem 13) of the family of all ends from a proximity space, a result which complements Mrówka's characterization [9] of the class of all clusters.

2. The dual to a proximity relation. The dual to a proximity relation can be characterized by the following system of axioms (compare with $[2 ; 3 ; 10])$ :

(2.1) If $A \ll B$, then $-B \ll-A$.

(2.2) $A \ll E$ for all $E$ if and only if $A=\varnothing$.

(2.3) If $A \ll B$ and $C \ll D$, then $A \cup C \ll B \cup D$.

(2.4) Given $A \ll C$, there exists $B$ such that both $A \ll B$ and $B \ll C$.

(2.5) If $x$ and $y$ are distinct points in $X$, then $x \ll-y$.

A completely regular topology is induced in $X$ by defining $E$ to be a neighborhood of $x$ whenever $x \ll E$. (See [3 and 10].) For later use we

Received by the editors May 15, 1961. 
list without proof some immediate results of the axiom system (2.1) $-(2.5)$.

From (2.1) and (2.3) we get

Theorem 1. If $A \ll B$ and $C \ll D$, then $A C \ll B D$.

From (2.2) and (2.3) we obtain

Theorem 2. If $A \ll \varnothing$, then $A=\varnothing$.

Using (2.4), (2.1), and the preceding theorems we get

Theorem 3. If $A \ll B$, then $A \subseteq B$.

A proximity space is discrete if the converse to Theorem 3 holds. From (2.1), (2.2), and (2.3) we obtain

Theorem 4. If $A \subseteq B, B \ll C$, and $C \subseteq D$, then $A \ll D$.

From the two preceding theorems comes

Theorem 5. If $A \ll B$ and $B \ll C$, then $A \ll C$.

Finally, using (2.4), (2.1), and Theorem 4 we get

Theorem 6. If $A \ll C$, then $\bar{A} \ll C$.

For $\mathcal{G}$ a class of subsets of a proximity space $X$ we define the following related classes of subsets of $X$. Let $\mathcal{G}^{0}$ be the class of all uniform neighborhoods of members of $g$ :

(2.6) $E$ is in $\mathcal{G}^{0}$ if and only if there exists $A$ in $\mathcal{G}$ with $A \ll E$.

Let $\mathcal{G}^{\prime}$ be the class of all sets which are close to every member of $\mathcal{G}$. Finally, let $\mathcal{G}^{*}$ be the dual of $\mathcal{G}$ :

(2.7) $E$ is in $\mathcal{G}^{*}$ if and only if $-E$ is not in $g$.

Theorem 7. $\mathcal{G}^{0}$ and $\mathcal{G}^{\prime}$ are dual classes.

Proof. We need only exhibit the following chain of equivalent statements.

(i) $E$ is in $\mathcal{G}^{0}$.

(ii) $A \ll E$ for some $A$ in $\mathrm{g}$.

(iii) $-E$ is remote from $A$ in $\mathcal{G}$.

(iv) $-E$ is not in $\mathcal{G}^{\prime}$.

3. Ends. We define an end from a proximity space $X$ to be a class $\mathcal{F}$ of subsets of $X$ satisfying the following two conditions:

(3.1) Given $B$ and $C$ in $\mathcal{F}$, there exists a nonempty set $A$ in $\mathcal{F}$ with both $A \ll B$ and $A \ll C$.

(3.2) If $A \ll B$, then either $-A$ or $B$ is in $F$. 
A nonvoid class $\mathcal{F}$ of subsets of $X$ which satisfies (3.1) will be called a uniform funnel. Clearly, the null set $\varnothing$ cannot belong to a uniform funnel. Also, since by (2.2) we have $\varnothing \ll X,(3.2)$ implies that $X$ is in every end from $X$. Thus there are no ends from an empty proximity space.

THEOREM 8. Every end is a filter.

Proof. We must show that an end $\mathcal{F}$ satisfies the following two conditions which characterize filters.

(3.3) $\mathcal{F}$ is a (discrete) funnel: $\mathcal{F}$ is a nonvoid class such that given $B$ and $C$ in $\mathcal{F}$, there exists a nonempty $A$ in $\mathcal{F}$ with both $A \subseteq B$ and $A \subseteq C$.

(3.4) $\mathcal{F}$ is closed under superset formation: If $C$ is in $\mathcal{F}$ and $D$ contains $C$, then $D$ is in $\mathcal{F}$.

Since $X$ is in every end, $\mathcal{F}$ is nonvoid. Th us (3.3) follows from (3.1) and Theorem 3. Given the hypothesis in (3.4), set $B=C$ in (3.1) yielding $A$ in $\mathcal{F}$ with $A \ll C$. Setting $B=A$ in Theorem 4 yields $A \ll D$. By (3.2) either $-A$ or $D$ is in $\mathscr{F}$. Since $A$ is in $\mathcal{F},(3.1)$ implies that $-A$ cannot be in $F$. Hence $D$ is in $F$. So (3.4) holds.

To show the equivalence between our definition of end and the definition given by Alexandroff [2] we first prove two lemmas.

LEMMA I. Every end is a maximal uniform funnel.

Proof. Let $\mathcal{F}$ be an end. So $\mathcal{F}$ is a uniform funnel. Let $\mathcal{G}$ be a uniform funnel containing $\mathfrak{F}$. We must show that $\mathrm{g}$ is contained in $\mathfrak{F}$.

Let $B$ be any member of $g$. By (3.1) there exists a nonempty set $A$

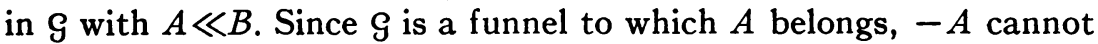
belong to $\mathcal{G}$. Since $\mathcal{G}$ contains $\mathcal{F},-A$ cannot belong to $\mathcal{F}$. By (3.2) $B$ belongs to $F$.

Lemma II. Let $\mathcal{F}$ be a uniform funnel and let $A \ll B$. If $A$ meets every member of $\mathcal{F}$, then $B$ belongs to some uniform funnel containing $\mathcal{F}$.

Proof. Let $\mathrm{g}$ be the class of all intersections with $A$ by members of $\Im$. We contend that $g^{0}$ is a uniform funnel, has $B$ as a member, and contains $\mathcal{F}$.

To prove $\mathcal{G}^{0}$ is a uniform funnel, let $P$ and $Q$ belong to $\mathcal{G}^{0}$. By the definition of $\mathcal{G}^{0}$ there exist $C$ and $D$ in $\mathcal{F}$ such that $A C \ll P$ and $A D \ll Q$. Since $\mathcal{F}$ is a funnel, there exists $E$ in $\mathcal{F}$ such that $E$ is contained in both $C$ and $D$. By Theorem $4, A E \ll P$ and $A E \ll Q$. By Theorem 1, $A E \ll P Q$. Using (2.4), choose $R$ such that $A E \ll R \ll P Q$. Then, since $A E$ belongs to $\mathcal{G}, R$ belongs to $\mathcal{G}^{0}$. Moreover, since $A E$ is nonempty by hypothesis, $R$ is nonempty by Theorem 3. Finally, by 
Theorem 4, $R \ll P$ and $R \ll Q$. So (3.1) holds for $\mathcal{G}^{\circ}$.

Take any $E$ in $F$. Then, since $E \ll X$ and $A \ll B$, Theorem 1 yields $A E \ll B$. So $B$ is in $\mathcal{G}^{0}$.

Given $D$ in $\mathcal{F}$, choose $E$ in $\mathcal{F}$ with $E \ll D$. By Theorem 4, $A E \ll D$. So $D$ is in $\mathcal{G}^{0}$. That is, $\mathcal{F}$ is a subclass of $\mathcal{G}^{0}$.

THEOREM 9. $\mathcal{F}$ is an end if and only if $\mathcal{F}$ is a maximal uniform funnel.

Proof. We need only prove the converse to Lemma I. Let $\mathcal{F}$ be a maximal uniform funnel and $A \ll B$ with $B$ not in $F$. We must show that $-A$ is in $F$.

Since $\mathcal{F}$ is maximal, Lemma II implies the existence of $E$ in $\mathcal{F}$ such that $A E$ is empty. Thus $-A$ contains a member $E$ of $F$. Hence, since $\mathcal{F}$ is maximal, Theorem 4 implies that $-A$ is in $\mathcal{F}$.

Since (3.1) is a property of finite character for $\mathcal{F}$, every uniform funnel can be extended to a maximal uniform funnel by the axiom of choice. Hence Theorem 9 yields

CoROllary 9a. Every uniform funnel is a subclass of some end.

\section{Duality between ends and clusters.}

TheOREM 10. $\mathcal{F}$ is an end if and only if $\mathcal{F}^{*}$ is a cluster. For discrete proximity spaces both ends and clusters are just ultrafilters.

Proof. From the definition of cluster given in [7] we obtain mutatis mutandis the following characterization of a cluster dual. $\mathcal{F}^{*}$ is a cluster if and only if its dual $\mathcal{F}$ satisfies the three conditions:

(a) If $A \ll B$, then either $-A$ or $B$ is in $F$.

(b) Given $B$ in $\mathcal{F}$, there exists $E$ with $-E$ not in $\mathcal{F}$ such that $E \ll B$.

(c) If $A$ and $B$ are in $\mathcal{F}$, then $A B$ is in $\mathcal{F}$.

Note that (a) is exactly (3.2).

Let $\mathcal{F}$ be an end. Then (c) follows from Theorem 8. To prove (b) take $A=E$ in (3.1). Then, since $E$ is in $\mathcal{F}$ and $\mathcal{F}$ is a uniform funnel, $-E$ is not in $\mathcal{F}$. Hence (b). Thus, (3.1) and (3.2) imply (a), (b), and (c).

Conversely, let $\mathcal{F}^{*}$ be a cluster. That is, let $\mathcal{F}$ satisfy (a), (b), and (c). Since (a) is just (3.2), we need only derive (3.1). Moreover, in view of (c) and Theorem 4 , we need only prove

(4.1) Given $B$ in $\mathcal{F}$, there exists a nonempty $A$ in $\mathcal{F}$ with $A \ll B$.

Given (b), use (2.4) to get $A$ such that $E \ll A \ll B$. Since $E \ll A$ and

$-E$ is not in $F$, (a) implies $A$ is in $F$. Since $\varnothing \ll X$, (a) implies $X$ is in F. Therefore, $E$ is nonempty. So Theorem 3 implies $A$ is nonempty. Hence (4.1).

The second part of Theorem 10 follows because an ultrafilter is 
self-dual and is just a maximal discrete funnel.

In view of Theorem 7, Theorem 10 yields

Corollary 10a. $\mathrm{G}^{0}$ is an end if and only if $\mathrm{g}^{\prime}$ is a cluster.

5. Applications of the duality theorem. It is now easy to establish a general relationship between ends and ultrafilters.

THEOREM 11. Every ultrafilter $\mathcal{F}$ from a proximity space contains a unique end $\mathcal{F}^{0}$.

Proof. Let $F$ be an ultrafilter. In Theorem 6 of [8] we proved that $\mathcal{F}^{\prime}$ is a unique cluster containing $\mathcal{F}$. Taking duals, $\mathcal{F}^{*}$ is a subclass of $\mathcal{F}^{*}$. Using Theorem 7 and the self-duality of $\mathcal{F}$, this reduces to the statement that $\mathcal{F}^{0}$ is a subclass of $\mathcal{F}$. That $\mathcal{F}^{0}$ is an end follows from Corollary $10 \mathrm{a}$.

Now let $\mathcal{G}$ be any end contained in $\mathcal{F}$. Then $\mathcal{G}^{0}$ is a subclass of $\mathcal{F}^{0}$. But $\mathcal{G}^{0}$ is just $\mathcal{G}$, since $\mathcal{G}$ is an end. So $\mathcal{G}$ is a subclass of $\mathcal{F}^{0}$. By Theorem $9, \mathfrak{F}^{0}$ is identical with $\mathcal{G}$. Hence, $\mathfrak{F}^{0}$ is unique.

THEOREM 12. $A \ll B$ in a proximity space $X$ if and only if every end from $X$ has either $-A$ or $B$ as members.

Proof. One implication is just (3.2). To prove the converse suppose $A$ is not uniformly interior to $B$. Then $A$ is close to $-B$. By Theorem 1 of [7] both $A$ and $-B$ belong to some cluster $\mathcal{F}^{*}$. Hence, both $-A$ and $B$ fail to belong to the dual class $\mathcal{F}$. By Theorem 10, $\mathcal{F}$ is an end.

ThEOREM 13. Let $\Phi$ be a family of filters (which we shall call " $\Phi$ filters") from a set $X$. In order that $\Phi$ be the family of all ends for some proximity relation, it is necessary and sufficient that the following four conditions hold:

(5.1) If $\mathcal{F}$ is $a \Phi$-filter, then the intersection of all sets in $\mathcal{F}$ has at most one point.

(5.2) If $\mathcal{F}$ is a $\Phi$-filter and $B$ belongs to $\mathcal{F}$, then there exists $a$ set $A$ in $\mathcal{F}$ such that every $\Phi$-filter has either $-A$ or $B$.

(5.3) If every $\Phi$-filter has at least one of the sets $D$ or $C$, then there exists a set $B$ such that every $\Phi$-filter which fails to have $D$ has $B$ and every $\Phi$-filter which fails to have $C$ has $-B$.

(5.4) Every ultrafilter from $X$ has some $\Phi$-filter as a subclass.

PROOF. Let $\Phi$ be the family of all ends for a given proximity relation. $\Phi$ is a family of filters by Theorem 8. Now (5.1) follows from (2.5) and (3.2). In view of Theorem 12, (5.2) follows from (3.1), while (5.3) is just (2.4) if we set $D=-A$. (5.4) follows from Theorem 11. 
So (5.1)-(5.4) are necessary for $\Phi$ to be the family of all ends for a given proximity relation.

To prove these conditions are sufficient, let $\Phi$ be a family of filters from $X$ such that (5.1)-(5.4) hold. Define

(5.5) $A \ll B$ if and only if every $\Phi$-filter has either $-A$ or $B$ as members.

Then (2.1) is trivial. Given $A \ll E$ for all $E$, then $A \ll \varnothing$. Since $\varnothing$ cannot belong to any filter, (5.5) implies that $-A$ belongs to every $\Phi$-filter. So $-A$ belongs to every ultrafilter from $X$, by (5.4). Hence $-A$ is $X$. So $A$ is $\varnothing$. Conversely, since $X$ belongs to every filter from $X, \varnothing \ll E$ for all $E$. Hence, (2.2).

Given the hypothesis of (2.3) in terms of (5.5), every $\Phi$-filter has either $-A$ or $B$ and also has either $-C$ or $D$. If a $\Phi$-filter $\mathcal{F}$ fails to have $-(A \cup C)$, which is just $(-A)(-C), \mathcal{F}$, being a filter, cannot have both $-A$ and $-C$. Hence, $\mathcal{F}$ must have either $B$ or $D$. So $\mathcal{F}$ must have $B \cup D$. Hence (2.3).

Now (2.4) is just (5.3) if we set $D=-A$ and apply (5.5). Similarly, (2.5) follows from (5.1) and (5.5).

Therefore, given (5.1)-(5.4), definition (5.5) induces a proximity relation.

That every $\Phi$-filter $\mathcal{F}$ is a uniform funnel follows from (5.2) and (5.5) since $\mathcal{F}$ is closed under finite intersections and we can apply Theorem 4. Since (3.2) follows from (5.5), every $\Phi$-filter is an end.

Finally, since every filter can be extended to an ultrafilter, Theorem 11 and (5.4) imply that every end from $X$ is a $\Phi$-filter.

\section{REFERENCES}

1. P. S. Alexandroff, On bicompact extensions of topological spaces, Mat. Sb. N.S. 5 (47) (1939) 403-423.

2. P. S. Alexandroff and V. Ponomarev, On bicompact extensions of topological spaces, Dokl. Akad. Nauk SSSR 121 (1958), 575-578.

3. V. A. Efremovich, The geometry of proximity, Mat. Sb. N.S. 31 (73) (1952), 189-200.

4. S. V. Fomin, Extensions of topological spaces, Ann. of Math. 44 (1953), 471-480.

5. H. Freudenthal, Kompaktisierungen und Bikompaktisierungen, Nederl. Akad. Wetensch Proc. Ser. A 54 (1951), 184-192.

6. - Neuaufbau der Endentheorie, Ann. of Math. 43 (1942), 261-279.

7. S. Leader, On clusters in proximity spaces, Fund. Math. 47 (1959), 205-213.

8. - On completion of proximity spaces by local clusters, Fund. Math. 48 (1960), 201-216.

9. S. Mrowka, Axiomatic characterization of the family of all clusters in a proximity space, Fund. Math. 48 (1960), 123-126.

10. Yu. M. Smirnov, On proximity spaces, Mat. Sb. N.S. 31(73) (1952), 543-574.

Rutgers, The State University 